Enferm Bras 2019;18(2):291-5

https://doi.org/10.33233/eb.v18i2.2449

\title{
RELATO DE CASO \\ Prevenção de lesão por pressão e o uso do colchão pneumático versus colchão piramidal em unidade de terapia intensiva
}

Alexandre Aguiar Pereira*, Ana Paula Figueiredo Montalvão França**, Andrea Fabiane Aguiar Chagas de Miranda ${ }^{* * *}$, Elaine Valéria Rodrigues ${ }^{* * *}$, Jaqueline Vieira Guimarães ${ }^{*}$, Maria Elizabete de Castro Rassy, D.Sc..****

*Enfermeiros Residentes em Atenção à Saúde da Mulher e da Criança da Universidade do Estado do Pará (UEPA/FSCMP), Belém/PA, ${ }^{* *}$ Enfermeira Especialista em Terapia Intensiva, Mestre em Gestão e Saúde na Amazônia (FSCMP), Belém/PA, ${ }^{* \star *}$ Enfermeira Especialista em Urgência e Emergência e Regulação em Serviços de Saúde, Mestre em Gestão e Saúde na Amazônia (FSCMP), Belém/PA, ${ }^{\star * \star \star E n f e r m e i r a ~ E s p e c i a l i s t a ~ e m ~ S a u ́ d e ~ d a ~ F a m i ́ l i a, ~ M e s t r e ~ e m ~}$ Gestão e Saúde na Amazônia (FSCMP), Belém/PA, ${ }^{* * * * * D o c e n t e ~ d e ~ G r a d u a c ̧ a ̃ o ~ e ~ P o ́ s-~}$ graduação da Universidade do Estado do Pará (UEPA), Belém/PA

Recebido em 8 de julho de 2018; aceito em 4 de abril de 2019.

Endereço para correspondência: Ana Paula Figueiredo Montalvão França, Rodovia Augusto Montenegro, 6955, Quadra 3, Casa 6, Parque Verde, 66635-110 Belém PA, E-mail: anapaula@alexandrohup.com; Alexandre Aguiar Pereira: alexandre_ap22@hotmail.com; Andrea Fabiane Aguiar Chagas de Miranda: andreaf_chagas@hotmail.com; Elaine Valéria Rodrigues: elainerik@gmail.com; Jaqueline Vieira Guimarães: jakque-k3@hotmail.com; Maria Elizabete de Castro Rassy: elizarassy50@hotmail.com

\section{Resumo}

Introdução: As lesões por pressão são injúrias na pele ou tecidos moles adjacentes, que normalmente encontram-se sobre uma proeminência óssea, consideradas como problema de saúde pública e fator de agravo para o paciente acamado. Objetivo: Relatar o caso de pacientes que utilizaram o colchão pneumático e/ou piramidal como estratégia de prevenção de lesões por pressão em Unidade de Terapia Intensiva. Métodos: Relato de caso, descritivo, realizado em uma Unidade de Terapia Intensiva Adulto de uma Maternidade de Referência na cidade de Belém/PA, em setembro de 2017, com 6 pacientes. Para a obtenção dos dados foi realizada a busca dos pacientes que utilizavam o colchão pneumático e/ou piramidal e anotações diárias de enfermagem. Resultados: $2(33,3 \%)$ dos pacientes que foram colocados sob o uso de colchão piramidal apresentaram lesão por pressão, enquanto dos $4(66,67 \%)$ que utilizaram o colchão pneumático, apenas $1(16,67 \%)$ paciente apresentou lesão. Conclusão: Sendo a prevenção de lesão por pressão uma das metas de Segurança do Paciente, concluiu-se que a utilização do colchão pneumático poderá contribuir para a redução do aparecimento dessas lesões em Unidade de Terapia Intensiva.

Palavras-chave: lesão por pressão, unidades de terapia intensiva, segurança do paciente.

\footnotetext{
Abstract

Pressure injury prevention and the use of air mattress versus convoluted foam mattress in intensive therapy unit

Introduction: Pressure injuries are damage caused on the skin or surrounding soft tissues which are typically found on prominent bones and are considered as a public health issue and an aggravating factor for the bedridden patient. Objective: To report the case of patients who made use of air mattress and/or convoluted foam mattress as a strategy for the prevention of pressure injury to Intensive Therapy Unit. Methods: Case report, descriptive, developed at an Adult Intensive Therapy Unit of a reference maternity hospital in Belém/PA, in September 2017, involving 6 patients. For the data acquisition, a search was performed for patients who utilize air mattress and/or convoluted foam mattress, as well as for nursing notes. Results: Two (33.3\%) of the patients who were assisted with the use of the convoluted foam mattress showed signs of pressure injury whereas, out of the $4(66.67 \%)$ of those who utilized the air mattress, only 1 $(16.67 \%)$ patient revealed signs of injury. Conclusion: Since pressure injury prevention is one of
} 
the purposes of the Patient Safety, it has been concluded that the use of the air mattress could contribute to the reduction of the emergence of these injuries in Intensive Therapy Unit.

Key-words: pressure injury, intensive therapy unit, patient safety.

\section{Resumen}

Prevención de lesiones por presión y el uso del colchón neumático versus colchón piramidal en la unidad de cuidados intensivos

Introducción: Lesiones por presión son heridas en la piel o tejidos blandos adyacentes que normalmente se encuentran en una proeminência de la estructura ósea, y se considera un problema de salud pública y factor agravante para los pacientes hospitalizados. Objetivo: Relatar el caso de pacientes que se han utilizado del colchón neumático y/o piramidal como estrategia de prevención de lesiones que se produzcan por la presión en Unidad de Cuidados Intensivos. Métodos: Descripción de caso realizado en la unidad de cuidados intensivos para adultos de una maternidad de referencia de la ciudad de Belém/PA, en septiembre del año 2017, con 6 pacientes. Los dados se obtuvieron através de una búsqueda a los pacientes que utilizaban el colchón neumático y/o piramidal, además de las anotaciones de enfermería. Resultados: Dos (33,3\%) de los pacientes que se sometieron al uso del colchón piramidal presentaron lesión por presión, mientras que los $4(66,67 \%)$ que utilizaron el colchón neumático, solamente 1 presentó. Conclusión: Siendo la prevención de lesión por presión una de las metas de seguridad al paciente, se concluye que la utilización del colchón neumático podría contribuir a la reducción de aparición de estas lesiones en Unidad de Cuidados Intensivos.

Palabras-clave: lesión por presión, unidades de cuidados intensivos, seguridad del paciente.

Introdução

As lesões por pressão (LPPs) são injúrias na pele ou tecidos moles adjacentes, que normalmente encontram-se sobre uma proeminência óssea ou causada por dispositivo médico ou outro artefato [1], consideradas como um problema de saúde pública e fator de agravo para o paciente internado, intensificando seu sofrimento, risco de mutilação, de infecção, prolongando o tempo de internação do mesmo, elevando a morbimortalidade, além de aumentar a carga de trabalho da equipe de enfermagem e os custos hospitalares [2].

Desta forma, os pacientes internados em Unidades de Terapia Intensiva (UTI) apresentam elevado risco para o desenvolvimento destas lesões, visto que apresentam restrição de mobilidade no leito e percepção sensorial diminuída por ação de sedativos e relaxantes musculares [3].

Nos últimos anos, a prevalência das LPPs vem aumentando, devido ao envelhecimento da população e consequentemente das doenças crônicas e lentamente debilitantes, que geram hospitalização prolongada [4]. Apesar da existência de vários estudos mundiais, estudos realizados no Brasil sobre a incidência deste evento adverso mostram números que variam entre $20 \%$ a $59 \%$, dependendo da população estudada [5].

A fim de garantir a segurança dos pacientes em Instituições de saúde, o Ministério da Saúde (MS) instituiu o Programa Nacional de Segurança do Paciente (PNSP), lançando protocolos de prevenção de incidentes, entre eles as LPPs. Nesse contexto, ações preventivas e sistematizadas da equipe multiprofissional e principalmente da equipe de enfermagem com o objetivo de diminuir a ocorrência destas lesões são de grande importância, gerando qualidade na assistência prestada [6].

A literatura tem apontado que a utilização de colchão pneumático, embora exija um maior investimento, pode gerar uma economia hospitalar com a redução da utilização de curativos industrializados e diminuição do tempo de internação [7], diferentemente do colchão piramidal ou "caixa de ovo" que deve ser trocado sempre que o colchão perder densidade ou altura, para não ter sua função anulada ou reduzida [8].

Diante do exposto, definiu-se como questão de pesquisa deste estudo: Como a utilização do colchão mais apropriado pode contribuir para a prevenção do aparecimento de lesões por pressão em pacientes acamados em Unidade de Terapia Intensiva?

Desta forma, este estudo tem por objetivo: relatar o caso de pacientes que utilizaram o colchão pneumático e/ou colchão piramidal como estratégia de prevenção de lesões por pressão, a fim de identificar o mais adequado para pacientes internados em Unidade de Terapia Intensiva. 
Relato de caso, com abordagem descritiva, realizado em uma UTI Adulto de uma Maternidade de Referência no Estado do Pará, durante as atividades práticas do Programa de Residência Multiprofissional em Saúde da Mulher e da Criança, da Universidade do Estado do Pará (UEPA), no mês de setembro de 2017. Esta Unidade possui 10 leitos e uma demanda de pacientes adultos clínicos, cirúrgicos e mulheres com gestação de alto risco.

Para a obtenção dos dados foi realizada a busca nos impressos assistenciais e evoluções diárias de enfermagem dos pacientes internados, presentes nos prontuários da Unidade. Elencou-se como critérios de inclusão: pacientes com avaliação de risco alto para desenvolvimento de lesão de acordo com a Escala de Braden, internados há mais de $\mathbf{3 0}$ dias, independentes da idade e que utilizavam o colchão pneumático e/ou piramidal como medida preventiva de LPP. Assim, incialmente, foram analisados os 10 prontuários dos pacientes internados, contudo 6 casos se adequaram aos critérios de inclusão e, portanto, foram selecionados e incluídos no estudo.

As informações coletadas foram inseridas em uma planilha eletrônica no software Microsoft Office Excel, versão 2010, e os resultados estão descritos e apresentados na tabela abaixo.

Tabela I - Dados dos casos internados na Unidade de Terapia Intensiva sob uso de colchão pneumático e/ou piramidal, Belém/PA, 2018.

\begin{tabular}{|c|c|c|c|c|c|c|c|}
\hline Código & Idade & $\begin{array}{l}\text { Diagnósticos } \\
\text { médico }\end{array}$ & Região & $\begin{array}{l}\text { Tipo de } \\
\text { colchão }\end{array}$ & Braden & Risco & $\begin{array}{l}\text { Dias de } \\
\text { internação } \\
\text { na UTI }\end{array}$ \\
\hline P.A.O.A. & 35 & $\begin{array}{l}\text { SIDA/neurotoxo- } \\
\text { plasmose/ } \\
\text { pneumocistose/ } \\
\text { traqueite/IRPC }\end{array}$ & $\begin{array}{l}\text { Sacral/ } \\
\text { Maleolar } \\
\text { bilateral/ } \\
\text { Calcaneo } \\
\text { bilateral/ } \\
\text { Trocanter } \\
\text { esquerdo }\end{array}$ & Piramidal & 8 & Alto & 31 \\
\hline T.F.A. & 83 & $\begin{array}{l}\text { IRPAG/DPOC/ } \\
\text { pneumonia/ } \\
\text { Alzheimer }\end{array}$ & $\begin{array}{l}\text { Sacra } \\
\text { (estagio III) }\end{array}$ & Piramidal & 8 & Alto & 30 \\
\hline M.R.S.B. & 19 & $\begin{array}{l}\text { Neuropatia a } \\
\text { esclarecer } \\
\text { associada a } \\
\text { gestação/ } \\
\text { paraplegia/pós- } \\
\text { cesarea/ } \\
\text { desidratação } \\
\text { aguda/pneumonia/ } \\
\text { IRPAG/SEPSE }\end{array}$ & $\begin{array}{l}\text { Não houve } \\
\text { LPP }\end{array}$ & Pneumático & 12 & Alto & 112 \\
\hline M.I.M.C. & 43 & $\begin{array}{l}\text { Mioma } \\
\text { uterino/IRPAG/ } \\
\text { Sepse }\end{array}$ & $\begin{array}{l}\text { Não houve } \\
\text { LPP }\end{array}$ & Pneumático & 12 & Alto & 73 \\
\hline P.L.C.P. & 37 & $\begin{array}{l}\text { ICS/PÓS- } \\
\text { Cesárea/TEP/PAV/ } \\
\text { Infecção do trato } \\
\text { urinario/diabetes } \\
\text { mellitus }\end{array}$ & $\begin{array}{l}\text { Não houve } \\
\text { LPP }\end{array}$ & Pneumático & 12 & Alto & 40 \\
\hline E.R.M. & 61 & $\begin{array}{l}\text { Nefrolitiase/ } \\
\text { IRA/IRPG/ } \\
\text { Neuropatia a } \\
\text { esclarecer }\end{array}$ & $\begin{array}{l}\text { Ocipital } \\
\text { (estágio II) }\end{array}$ & Pneumático & 11 & Alto & 88 \\
\hline
\end{tabular}

Fonte: Dados do Estudo.

\section{Discussão}

Quanto ao tipo de colchão utilizado para prevenir as LPPs, $2(33,3 \%)$ pacientes foram colocados sob o uso de colchão piramidal e $100 \%$ apresentaram LPPs, enquanto dos $4(66,67 \%)$ que utilizaram o colchão pneumático, apenas $1(16,67 \%)$ paciente apresentou LPP. 
Estudo realizado em 2012, em Unidades Coronarianas de um Hospital Universitário de Pernambuco, mostrou redução da incidência de LPPs após implementação da utilização de colchão pneumático, à medida que este tipo de superfície gerou uma redução na incidência das LPPs de 55,4\%, comparando os anos de 2011 e 2012 [5].

Quanto a avaliação do risco para desenvolvimento de LPP realizado com o auxílio da Escala de Braden, $6(100 \%)$ dos pacientes foram classificados como de alto risco para 0 aparecimento de lesão, dos quais $3(50 \%)$ desenvolveram LPP.

Tais instrumentos avaliativos, como a escala de Braden, tornam-se de suma relevância para determinação do risco de um paciente, uma vez que os valores baixos mostram maior propensão para desenvolver estas lesões. A avaliação diária deste risco pelo profissional enfermeiro, possibilita-o implementar ações individualizadas que minimizem a incidência deste agravo [9].

Os 6 casos (100\%) estudados possuíam uma ou mais patologias de base, consideradas fatores de agravo para o aparecimento de LPPS, uma vez que a presença de comorbidades é considerado fator intrínseco no desenvolvimento de lesões cutâneas, principalmente as doenças crônicas, como diabetes e doenças cardiovasculares [10]. Essas enfermidades facilitam a imobilidade do paciente e exposição prolongada à umidade, má nutrição e capacidade reduzida de sentir dor e/ou pressão, condições que aumentam a probabilidade do surgimento de uma LPP [11].

Em relação ao tempo de internação hospitalar, todos os pacientes (100\%) permaneceram internados pelo menos 30 dias. Estudos apontam que quanto maior o tempo de internação de um paciente, maior é sua exposição aos fatores de risco para o desenvolvimento de LPPs [12], reforçando a necessidade de utilização do colchão mais adequado para pacientes com tempo de internação prolongado.

Fator relevante para o aparecimento de LPP refere-se à idade dos pacientes. Neste estudo, dos 3 pacientes que desenvolveram LPP, 1 era adulto jovem e 2 eram pacientes idosos, de acordo com a classificação etária da Organização Mundial da Saúde [13]. Nesse contexto, estudos demonstram que a idade avançada e o sexo masculino [14] são fatores preditivos para o aparecimento de LPPs.

Durante o período de estudo no setor, evidenciou-se a importância do trabalho da equipe de saúde, principalmente a de enfermagem, na prevenção de LPPs, por meio do estabelecimento do cuidado diário e prática baseada em evidências, tendo em vista que, em sua maioria, as lesões são evitáveis. Cabe à enfermagem, por meio da utilização de seus conhecimentos específicos, estabelecer metas, utilizar escalas preditivas de avaliação de risco e implantar medidas de prevenção e tratamento das lesões, adotando protocolos baseados em diretrizes internacionais e estabelecendo um processo avaliativo contínuo da integridade da pele [15].

Conclusão

Sendo a prevenção de LPPs uma das metas de Segurança do Paciente, concluiu-se que a utilização do colchão pneumático pode influenciar na redução dos riscos de aparecimento de LPPs em pacientes internados em UTI, possibilitando a prevenção adequada desse tipo de agravo.

Por esta razão, a utilização do colchão mais adequado pode trazer grandes benefícios para o paciente e reduzir a incidência de LPPs na Unidade, reforçando a necessidade de intensificação dos cuidados de toda equipe multiprofissional e implementação de protocolos que estimulem a utilização do colchão pneumático.

O estudo apresentou algumas limitações relacionadas a não inclusão da equipe multiprofissional de saúde do Hospital; à inclusão de apenas um setor da unidade hospitalar e a avaliação de uma amostra reduzida de casos e em um tempo limitado. Sugere-se, então, a realização de novos estudos sobre o assunto, utilizando-se uma amostra e tempo mais amplos e que reforcem os resultados encontrados.

Os resultados apontam para a necessidade de investimento na utilização de colchão pneumático em UTI, por parte das instituições hospitalares. Assim, espera-se contribuir para o conhecimento dos profissionais que atuam nessas Unidades e sensibilização destes para a importância de utilizarem o colchão pneumático na prevenção de LPP, a fim de subsidiar o planejamento e implementação desse cuidado na rotina do setor. 
1. National Pressure Ulcer Advisory Panel (NPUAP). National Pressure Ulcer Advisory Panel (NPUAP) announces a change in terminology from pressure ulcer to pressure injury and updates the stages of pressure injury. 13 Apr. 2016. [citado 2018 Abr 12]. Disponível em: http://www.npuap.org/national-pressure-ulcer-advisory-panel-npuapannounces-a-change-in-terminology-from-pressure-ulcer-to-pressure-injury-andupdates-the-stages-of-pressure-injury/

2. Bergquist-Beringer S, Gajewski B, Dunton N, Klaus $S$. The reliability of the National databaseof nursing quality indicators pressure ulcer indicator: A triangulation approach. J Nurs Care Qual 2011;26(4):292-301.

3. Wada A, Teixeira Neto N, Ferreira MC. Úlceras por Pressão. Rev Med 2010;89(3/4):170-7.

4. Wiethan NS, Silva GQ. Assistência de enfermagem na prevenção de lesões por pressão: revisão de literatura. Rev dos TCCs e Semanas Acadêmicas da ULBRA de Cachoeira do Sul 2016;1(1).

5. Pinho CM, Correia RN, Valença MP, Cavalcanti ATA, Gomes ET. Use of the air mattress in the reduction of pressure ulcers: efficacy and perceptions of nursing. J Nurs UFPE on line 2014;8(8):2729-35.

6. Ministério da Saúde (BR). Anvisa. Fiocruz. Anexo 2: Protocolo para prevenção de úlcera por pressão. Brasília: Ministério da Saúde; 09 de julho de 2013.

7. Luz SR, Lopacinski AC, Fraga R, Urban CA. Úlceras de Pressão. Geriatria \& Gerontologia 2010;4(1):36-43. https://doi.org/10.5205/reuol.6081-52328-1SM.0808201421

8. Lobato CP, Santos LF, Teixeira NGSC, Agostinho MR, Souza TS, Gomes KW, et al. TeleCondutas - Lesão por Pressão. TelessaúdeRS. Porto Alegre: Universidade Federal do Rio Grande do Sul; 2017.

9. Ascari RA, Veloso J, Silva OM, Kessler M, Jacoby AM, Schwaab G. Úlcera por pressão: um desafio para a enfermagem. Braz J Surg Clin Res 2014;6(1):11-6.

10. Silva DP, Barbosa MH, Araújo DF, Oliveira LP, MELO AF. Úlcera por pressão: avaliação de fatores de risco em pacientes internados em um hospital universitário. Rev Eletr Enf 2011;13(1):118-23.

11. Fernandes LM. Úlcera de pressão em pacientes críticos hospitalizados. Uma revisão integrativa da literatura [Dissertação]. São Paulo: Escola de Enfermagem de Ribeirão Preto/USP; 2000. 168 p.

12. Barbosa TP, Beccaria LM, Poletti NAA. Avaliação do risco de úlceras por pressão em UTI e assistência preventiva de enfermagem. Rev Enferm UERJ 2014;22(3):353-8.

13. World Health Organization (WHO). Active ageing - A police framework. A contribution of the World Health Organization to the second United Nations World Assembly on Aging. Madrid: Spain, April 2002. [citado 2018 Abr 25]. Disponível em: https://extranet.who.int/agefriendlyworld/wp-content/uploads/2014/06/WHO-ActiveAgeing-Framework.pdf

14. Moraes JT, Borges EL, Lisboa CR, Cordeiro DCO, Rosa EG, Rocha NA. Conceito e classificação de lesão por pressão: atualização no National Pressure Ulcer Advisory Panel. Rev Enferm Cent Oeste Min 2016;6(2):2292-2306.

15. Rogenski NMB, Kurcgant $P$. Incidência de úlceras por pressão após a implementação de um protocolo de prevenção. Rev Latinoam Enferm 2012;20(2). 